\title{
31. DATA REPORT: MOLECULAR AND STABLE ISOTOPE ANALYSES OF SORBED AND FREE HYDROCARBON GASES OF LEG 146, CASCADIA AND OREGON MARGINS ${ }^{1}$
}

\author{
Michael J. Whiticar ${ }^{2}$ and Martin Hovland ${ }^{3}$
}

\section{INTRODUCTION}

The collection and analysis of sediment gases, including light hydrocarbons, sulfur, and permanent gases, is a required routine during Ocean Drilling Program (ODP) drilling operations with the JOIDES Resolution. These measurements are a critical component of the shipboard safety and environmental protection conducted by the shipboard organic geochemists and ODP geochemistry technical staff. In addition, these gas analyses, in particular the hydrocarbons, are a valuable measurement asset for various scientific aspects of ODP investigations. This is because these gases are ubiquitous and diagnostic for many subsurface processes. For example, during Leg ODP 146 on the basis of determinations of the $\mathrm{C}_{1}-\mathrm{C}_{6}$ hydrocarbons (methane through hexane) it was possible to:

1. Differentiate between the occurrences of bacterial and thermogenic gases;

2. Identify autochthonous vs. allochthonous (i.e., migrated) gases;

3. Signal the approach and presence of free gases (e.g., associated with gas hydrate dissociation);

4. Pinpoint active fractures or fault zones or seepages with fluid and/or gas flow; and

5. Define the subsurface diagenetic and catagenetic regimes.

The shipboard characterization of the gases is based on concentration and molecular ratios, obtained by gas chromatography (GC). However, a more reliable classification of hydrocarbons found unexpectedly involves the shore-based determination of the stable carbon isotope ratios of the hydrocarbons, particularly methane. The combination of ${ }^{2} \mathrm{H} /{ }^{1} \mathrm{H}$ and ${ }^{13} \mathrm{C} /{ }^{12} \mathrm{C}$ ratios of methane are especially powerful at discriminating between bacterial, thermogenic, geothermal, hydrothermal, and abiogenic methane (Fig. 1). In concert with the molecular and concentration data, the ${ }^{13} \mathrm{C} /{ }^{12} \mathrm{C}$ ratios of light hydrocarbons can also identify secondary alterations such as mixing or microbial oxidation (Fig. 2).

Additional gas characterization with molecular and stable carbon isotopes is provided by differentiating (1) dissolved or free gases in the interstices of the sediment from (2) the gases bound or sorbed on the organics and minerals in the sediments. Over the past 15 years, the Bundesanstalt für Geowissenschaften und Rohstoffe (BGR-method) Hannover, FRG has pioneered the development of measurement methodologies and interpretative schemes to take advantage of the gas genetic information contained in both the free and sorbed phases in sediments (e.g., Faber and Stahl, 1984). The use of sorbed and free gas analyses has been applied successfully on several ODP legs, in-

'Carson, B., Westbrook, G.K., Musgrave, R.J., and Suess, E. (Eds.), 1995. Proc. ODP, Sci. Results, 146 (Pt. 1): College Station, TX (Ocean Drilling Program)

${ }^{2}$ School of Earth and Ocean Sciences, University of Victoria, P.O. Box 1700, Victoria, British Columbia V8W 2Y2, Canada.

Statoil, P.O. Box 300, N-4001, Stavanger, Norway.

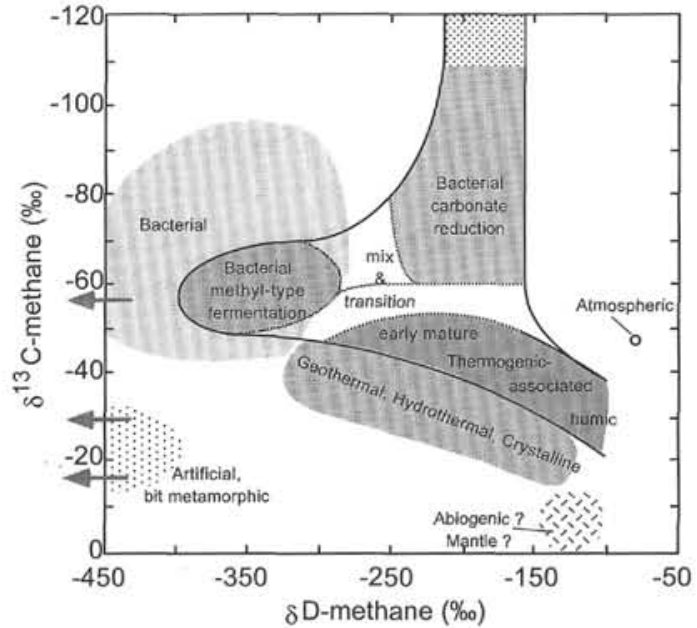

Figure 1. Gas classification diagram using the combination of stable carbon and hydrogen isotope ratios of methane (after Whiticar, 1990).

cluding Legs 104 (Whiticar and Faber, 1989), 112 (Whiticar and Suess, 1990), and 139 (Whiticar et al., 1994).

This is a data report of the light hydrocarbons encountered during drilling on the Cascadia and Oregon Margins. Some of this information is contained in the individual site chapters in the Initial Reports volume of Leg 146 (Westbrook, Carson, Musgrave, et al., 1994). Here, we document and make available our new shore-based data, which include:

1. Ratios of ${ }^{13} \mathrm{C} /{ }^{12} \mathrm{C}$ methane for selected Vacutainer ${ }^{(1)}$ samples (free gas);

2. Development of a new sorbed/total gas extraction apparatus, in conjunction with this ODP Leg 146 investigation;

3. Concentrations of total methane in sediments by acid-vacuum gas desorption; and

4. Ratios of ${ }^{13} \mathrm{C} /{ }^{12} \mathrm{C}$ total methane from desorbed sediment samples.

Detailed interpretation and reporting of these and other geochemical results will be provided in the companion synthesis paper of Whiticar et al. (this volume).

\section{METHODS}

The techniques used to collect and analyze the sediment gas during shipboard operations are described in Shipboard Scientific Party (1994, p. 30). These will be presented briefly here, along with a description of the new mechanical acid-vacuum gas desorption device for sorbed/total gas analysis. 
Figure 2. Three-dimensional natural gas classification diagram using the combination of stable carbon isotope ratios of methane, molecular $\mathrm{C}_{1} /\left(\mathrm{C}_{2}+\mathrm{C}_{3}\right)$ ratios, and gas concentration (after Whiticar, 1990).

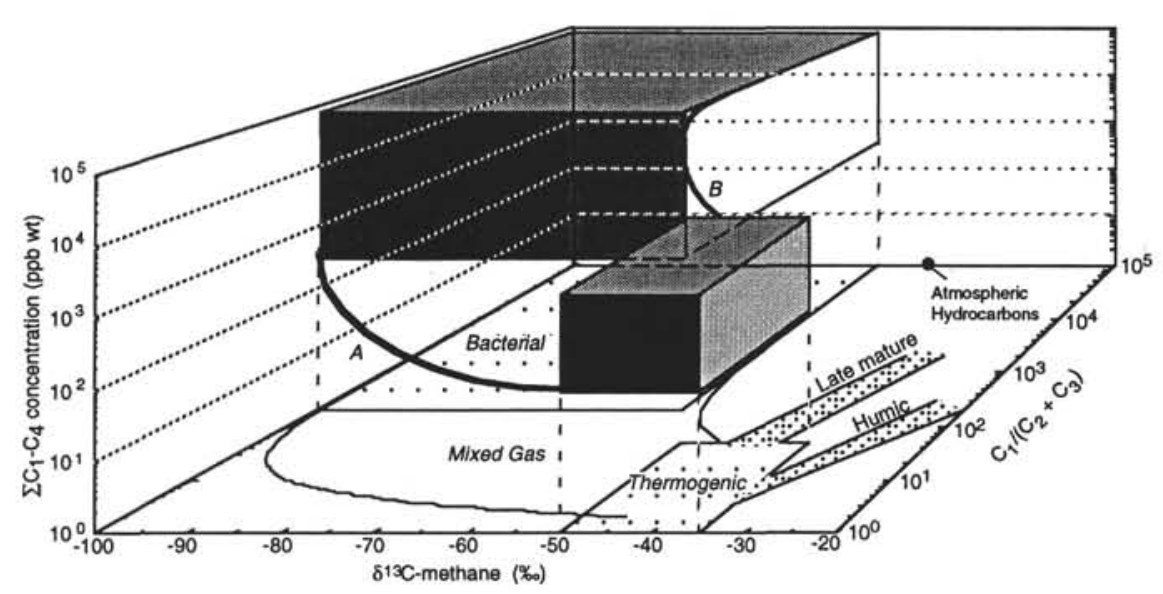

\section{Sampling and Measurement for Headspace and Vacutainer Gases}

During Leg 146, the compositions and concentrations of hydrocarbons and other gases were monitored in the sediments generally at intervals of one per core. Briefly, the two methods used are:

1. Headspace (HS) method:

a. 5-mL sediment core taken with a cork borer and sealed into a serum vial;

b. vial warmed to $60^{\circ} \mathrm{C}$;

c. aliquots of gas released by the sediments analyzed by GC.

2. Vacutainer (V) or expansion void gas (EVG) method:

a. samples taken on catwalk into pre-evacuated glass Vacutainers when gas pockets or expansion voids occurred in cores as they arrived on deck;

b. aliquots of gas analyzed by GC.

\section{Gas Chromatographic Systems}

The two systems used were the (1) Hach-Carle AGC Series 100 (Model 211), a standard packed column isothermal flame ionization detector (FID) GC, attached to Hewlett-Packard Model 3393A Integrator; and (2) the Hewlett-Packard 5890A Natural Gas Analyzer, modified by John Booker \& Company, Austin, Texas, modified multivalve and multicolumn, temperature-programmed GC equipped with both thermal conductivity (TCD) and FID, and two HewlettPackard Model 3393A Integrators dedicated to the TCD and FID.

\section{Sampling and Measurement for Total and Sorbed Gases}

Sediment samples for the sorbed and total hydrocarbons were obtained from the sediments collected for interstitial fluids (see Shipboard Scientific Party, 1994, p. 32). The sediment was frozen immediately after subsectioning and stored frozen until analysis.

The previous BGR-method used to desorb gases from sediments required 100-200 of sediment and a bulky glass vacuum extraction line (Faber and Stahl, 1984). This was necessary in order to obtain at least $50 \mathrm{nmol}$ of $\mathrm{CH}_{4}$ for the ${ }^{13} \mathrm{C} /{ }^{12} \mathrm{C}$ determination by conventional isotope ratio mass spectroscopy (IRMS). With the development of the on-line, gas chromatograph/combustion/isotope ratio mass spectrometer (GC/C/IRMS) (Fig. 3), we are now able to measure ${ }^{13} \mathrm{C} /{ }^{12} \mathrm{C}$ on extremely small quantities of methane ( 100 picomole, Whiticar and Cederberg, in press). This reduction in the amount of gas required also reduced the amount of sediment need to be degassed, and hence the degassing apparatus. Details of the new method are reported by Whiticar (in press).

Figure 4 shows the new sorbed gas desorption device. It consists of a thick-walled glass reaction vessel, with a three-port lid sealed by an O-ring/clamp. The lid provides for a transducer measurement of pressure/vacuum, and a valve-septum arrangement for addition of solutions and removal of gas samples. The procedure is briefly described below.

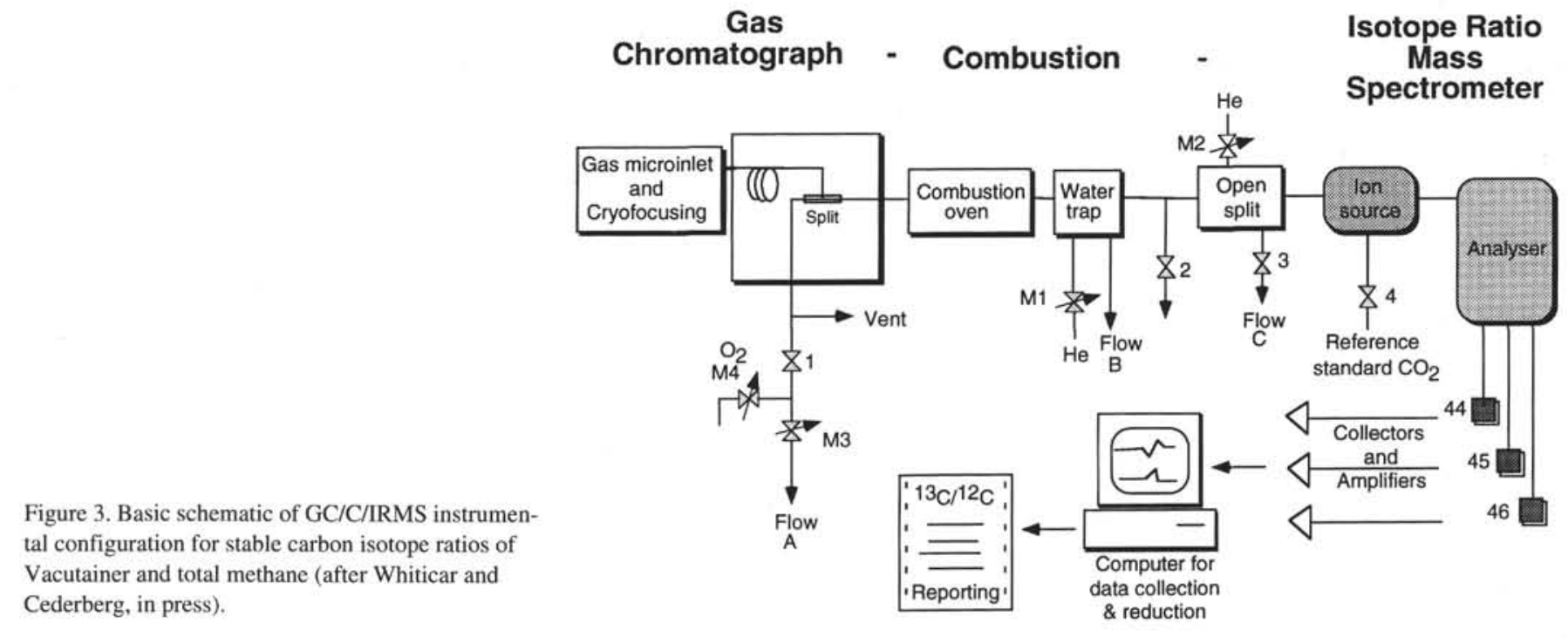




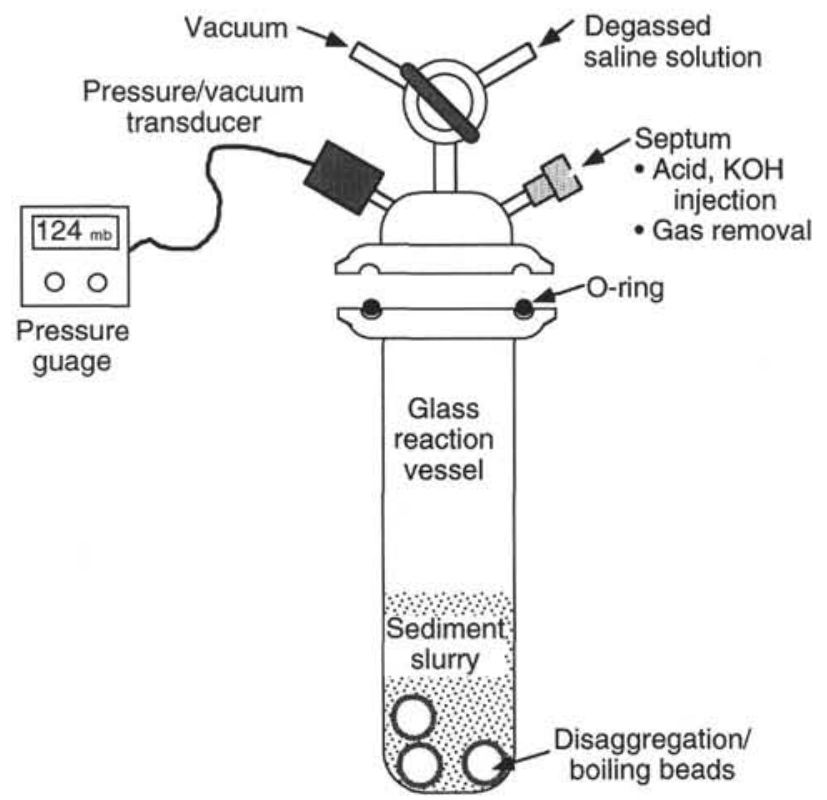

Figure 4. Light hydrocarbon acid/vacuum sediment gas desorption device (äter Whiticar, in press).

A known weight of frozen sediment, typically $1-5 \mathrm{~g}$ wet sediment, is thawed under vacuum in the apparatus. Enough saline solution (degassed water saturated with $\mathrm{NaCl}$ ) is added to make a slurry using a vortex mixer. Phosphoric acid is added slowly to release the sorbed gas fraction into the headspace. At the same time some carbonates will dissolve and generate free $\mathrm{CO}_{2}$. It is the carbonate content of the sediment, and hence amount of $\mathrm{CO}_{2}$ released that limits the practical sample size. The vessel is immersed in a water bath and the slurry is brought to a boil under reduced pressure $\left(75^{\circ} \mathrm{C}, 200 \mathrm{mbar}\right)$. $\mathrm{KOH}$ is added to precipitate the $\mathrm{CO}_{2}$, leaving the desorbed hydrocarbons and some $\mathrm{N}_{2}, \mathrm{O}_{2}, \mathrm{CO}_{2}, \mathrm{H}_{2} \mathrm{O}$, and possibly $\mathrm{H}_{2} \mathrm{~S}$ in the headspace of the reaction vessel. The vessel is brought to atmospheric pressure with degassed saline solution. Aliquots of gas are taken for analysis. Gases are then analyzed by conventional GC or by GC/C/IRMS. At our current stage of the method development, it is still possible that a portion of the free gas could be contained in the sorbed gas fraction. Because of this, we refer to this analysis at this stage as a total gas analyses. Continued evolution of this method will effectively eliminate the free gas fraction.

\section{Concentration Units and Notation}

The gases measured by the headspace and Vacutainer/EVG methods are reported on a gas volumetric basis, in parts per million by volume (ppmv), e.g., $\mu \mathrm{l} \mathrm{CH}_{4} / \mathrm{L}$ sample. In the case of the Vacutainer/ EVG the partial pressures of the gases measured are similar to those in the gas pocket of the sediment core liner. Inherent in the sampling for the headspace measurement, there is considerable contamination of air in the vial prior to sealing. Hence, the abundances are only proportional or relative. The concentration of methane in the sorbed gases is reported on a wet-sediment weight basis, i.e., $\mu \mathrm{g} \mathrm{CH}_{4} / \mathrm{kg}$ wet sediment (ppb wt).

\section{Stable Isotope Notation}

For practical reasons, stable isotope data are determined as a ratio, for example, ${ }^{13} \mathrm{C} /{ }^{12} \mathrm{C}$, rather than as an absolute molecular abundance, and are reported as the magnitude of excursion in per mil of the sam- ple isotope ratio relative to a known standard isotope ratio. The usual $\delta$-notation generally used in the earth sciences is:

$$
\delta^{13} \mathrm{C}(\% o)=\frac{{ }^{13} \mathrm{C} /{ }^{12} \mathrm{C} \text { sample }-\left({ }^{13} \mathrm{C}\right) /{ }^{12} \mathrm{C} \text { standard }}{{ }^{13} \mathrm{C} /{ }^{12} \mathrm{C} \text { standard }} \times 10^{3}
$$

where the ${ }^{13} \mathrm{C} /{ }^{12} \mathrm{C}$ isotope ratio is referenced relative to the PDB standard.

\section{RESULTS}

Only the basic analytical results are presented in the following tables and figures. Readers interested in interpretative aspects and discussion regarding the data should refer to Whiticar et al. (in press and this volume), and Hovland et al. (this volume).

\section{Hole 888B, Cascadia Margin}

The results of the headspace, Vacutainer, and total gas analyses for Hole 888B are presented in Tables 1-3, respectively. Representative depth plots for the methane concentrations and $\delta^{13} \mathrm{C}_{\text {methane }}$ are shown in Figures 5 and 6.

\section{Hole 889A, Cascadia Margin}

The results of the headspace, Vacutainer, and total gas analyses for Hole 889A are presented in Tables 4-6, respectively. Representative depth plots for the methane concentrations and $\delta^{13} C_{\text {methane }}$ are shown in Figures 7 and 8.

\section{Hole 891B, Oregon Margin}

The results of the headspace, Vacutainer, and total gas analyses for Hole 891B are presented in Tables 7 and 8. Representative depth plots for the methane concentrations and $\delta^{13} \mathrm{C}_{\text {methane }}$ are shown in Figures 9 and 10 .

\section{Site 892, Oregon Margin}

The results of the headspace, Vacutainer, and total gas analyses for Site 892 are presented in Tables 9-11, respectively. Representative depth plots for the methane concentrations and $\delta^{13} \mathrm{C}_{\text {methane }}$ are shown in Figures 11 and 12.

\section{ACKNOWLEDGMENTS}

We would like to thank F. Harvey-Kelly for the desorption laboratory work and T. Cederberg for operation of the GC/C/IRMS. The sorbed gas analyses were supported by a Nordic Research grant to M.H. The GC/C/IRMS facility was funded largely by a grant to M.J.W. by NSERC Strategic (STR0118459) and Operating (OPG0105389) Grants.

\section{REFERENCES}

Faber, E., and Stahl, W., 1984. Geochemical surface exploration for hydrocarbons in North Sea. AAPG Bull., 68:363-386.

Shipboard Scientific Party, 1994. Explanatory notes. In Westbrook, G.K., Carson, B., Musgrave, R.J., et al., Proc. ODP, Init. Repts., 146 (Pt. 1); College Station, TX (Ocean Drilling Program), 15-48.

Westbrook, G.K., Carson, B., Musgrave, R.J., et al., 1994. Proc. ODP, Init. Repts., 146 (Pt. 1): College Station, TX (Ocean Drilling Program).

Whiticar, M.J., 1990. A geochemical perspective of natural gas and atmospheric methane. In Durand, B., and Behar, F. (Eds.), Advances in 
Organic Geochemistry 1989, Part I. Organic Geochemistry in Petroleum Exploration. Org. Geochem., 16:531-547.

, in press. An acid/vacuum micro-extraction device for molecular and stable isotopic analysis of free and sorbed sediment gases. Anal. Chem.

Whiticar, M.J., and Cederberg, T., in press. Stable carbon isotope determinations of hydrocarbon gases at the picomole level by GC-C-IRMS. Anal.Chem.

Whiticar, M.J., and Faber, E., 1989. Molecular and stable isotope composition of headspace and total hydrocarbon gases at ODP Leg 104, Sites 642, 643, and 644, Vøring Plateau, Norwegian Sea. In Eldholm, O., Thiede, J., Taylor, E., et al., Proc. ODP, Sci. Results, 104: College Station, TX (Ocean Drilling Program), 327-334.
Whiticar, M.J., Faber, E., Whelan, J.K., and Simoneit, B.R.T., 1994. Thermogenic and bacterial hydrocarbon gases (free and sorbed) in Middle Valley, Juan de Fuca Ridge, Leg 139. In Mottl, M.J., Davis, E.E., Fisher, A.T., and Slack, J.F. (Eds.), Proc. ODP, Sci. Results, 139: College Station, TX (Ocean Drilling Program), 467-477.

Whiticar, M.J., and Suess, E., 1990. Characterization of sorbed volatile hydrocarbons from the Peru margin, Leg 112, Sites 679, 680/681, 682, 684, and 686/687. In Suess, E., von Huene, R., et al., Proc. ODP, Sci. Results, 112: College Station, TX (Ocean Drilling Program), 527-538.

Date of initial receipt: 12 September 1994

Date of acceptance: 12 April 1995

Ms 146SR-212

Table 1. Headspace analyses for Hole 888B.

\begin{tabular}{|c|c|c|c|c|c|c|c|c|c|}
\hline $\begin{array}{l}\text { Core, section, } \\
\text { interval }(\mathrm{cm})\end{array}$ & $\begin{array}{l}\text { Depth } \\
\text { (mbsf) }\end{array}$ & $\mathrm{O}_{2}$ & $\mathrm{~N}_{2}$ & $\mathrm{CH}_{4}$ & $\mathrm{CO}_{2}$ & $\mathrm{C}_{2} \mathrm{H}_{6}$ & $\mathrm{C}_{3} \mathrm{H}_{6}$ & $\mathrm{C}_{1} / \mathrm{C}_{2}$ & $\mathrm{C}_{1} / \mathrm{C}_{2+}$ \\
\hline $1 \mathrm{H}-1,5-55$ & 0.3 & 62 & 380 & 1.2 & 373 & & & $1 \mathrm{E}+6$ & $1 E+6$ \\
\hline $1 \mathrm{H}-1,58-108$ & 0.8 & 65 & 402 & 0.9 & 158 & & & $1 E+6$ & $1 E+6$ \\
\hline $1 \mathrm{H}-1,111-161$ & 1.4 & 1,490 & 1,388 & 2.0 & 433 & & & $1 \mathrm{E}+6$ & $1 \mathrm{E}+6$ \\
\hline $1 \mathrm{H}-2,164-214$ & 3.4 & 70 & 428 & 1.4 & 2 & & & $1 \mathrm{E}+6$ & $1 \mathrm{E}+6$ \\
\hline $1 \mathrm{H}-3,0-5$ & 3 & 27,736 & 116,760 & 3.2 & 4,954 & & & $\mathrm{IE}+6$ & $1 \mathrm{E}+6$ \\
\hline $1 \mathrm{H}-3,50-53$ & 3.5 & 18,479 & 80,024 & 3.8 & & & & $1 \mathrm{E}+6$ & $1 \mathrm{E}+6$ \\
\hline $2 \mathrm{H}-4,50-53$ & 11 & 2,534 & 4,991 & 6.2 & 3,733 & & & $\mathrm{IE}+6$ & $1 E+6$ \\
\hline $2 \mathrm{H}-6,0-5$ & 13 & 6,862 & 31,144 & 4.1 & 95 & & & $1 \mathrm{E}+6$ & $1 E+6$ \\
\hline $3 \mathrm{H}-5,0-5$ & 21 & 4,330 & 16,148 & 3.3 & 1,385 & & & $1 \mathrm{E}+6$ & $1 E+6$ \\
\hline $4 \mathrm{H}-5,0-5$ & 31 & 2,536 & 4,803 & 4.0 & 96 & & & $1 E+6$ & $1 \mathrm{E}+6$ \\
\hline $5 \mathrm{H}-5.0-5$ & 40 & 93 & 568 & 2.7 & 3 & & & $1 \mathrm{E}+6$ & $1 E+6$ \\
\hline $6 \mathrm{H}-6,0-5$ & 51 & 3,622 & 8.709 & 1.5 & 9 & & & $1 \mathrm{E}+6$ & $1 E+6$ \\
\hline $7 \mathrm{H}-5,0-5$ & 59 & 2,459 & 4,224 & 4.4 & 52 & & & $1 \mathrm{E}+6$ & $1 \mathrm{E}+6$ \\
\hline $8 \mathrm{H}-5,0-5$ & 69 & 2,572 & 4,609 & 4.1 & & & & $1 \mathrm{E}+6$ & $1 \mathrm{E}+6$ \\
\hline $9 \mathrm{H}-4,0-5$ & 77 & 21,125 & 89,807 & 1.0 & 24,73 & & & $1 \mathrm{E}+6$ & $1 E+6$ \\
\hline $9 \mathrm{H}-4,147-150$ & 78 & 93 & 570 & 0.4 & 5 & & & $1 E+6$ & $1 \mathrm{E}+6$ \\
\hline $10 \mathrm{H}-5,0-5$ & 88 & 1.701 & 1,539 & 110.5 & 4,795 & & & $1 \mathrm{E}+6$ & $1 E+6$ \\
\hline $11 \mathrm{H}-2,0-5$ & 93 & 6,666 & 28,959 & 150.9 & 1.384 & & & $1 \mathrm{E}+6$ & $1 \mathrm{E}+6$ \\
\hline $11 \mathrm{H}-3,0-5$ & 94 & 1.557 & 1.402 & 91.2 & 5,156 & & & $1 \mathrm{E}+6$ & $1 \mathrm{E}+6$ \\
\hline $12 \mathrm{H}-3,0-5$ & 104 & 240.916 & & 4.4 & 9,014 & & & $1 \mathrm{E}+6$ & $1 E+6$ \\
\hline $13 \mathrm{H}-4,0-5$ & 113 & 13,635 & 59,395 & 0.8 & 82 & & & $1 E+6$ & $1 \mathrm{E}+6$ \\
\hline $14 \mathrm{H}-5,0-5$ & 124 & 302,313 & & 0.7 & 4,744 & & & $1 E+6$ & $1 \mathrm{E}+6$ \\
\hline $15 \mathrm{H}-3,0-5$ & 131 & 39,868 & 165,777 & 0.4 & 77 & & & $1 E+6$ & $1 E+6$ \\
\hline $16 \mathrm{H}-6,0-5$ & 145 & 1,313 & 1,224 & 0.7 & 1,279 & & & $1 \mathrm{E}+6$ & $1 \mathrm{E}+6$ \\
\hline $17 \mathrm{H}-5,0-5$ & 153 & 4,224 & 14,061 & 1.5 & 63 & & & $1 E+6$ & $1 \mathrm{E}+6$ \\
\hline $18 \times-5,0-5$ & 162 & 22.574 & 95,665 & 0.2 & & & & $1 E+6$ & $1 \mathrm{E}+6$ \\
\hline $19 \times-5,0-5$ & 172 & 1,633 & 2,566 & 0.3 & 493 & & & $1 E+6$ & $1 \mathrm{E}+6$ \\
\hline $20 X-1,0-5$ & 175 & 3.873 & 11.831 & 3.6 & 190 & & & $1 E+6$ & $1 \mathrm{E}+6$ \\
\hline $21 X-1,0-2$ & 185 & 245,792 & & 0.3 & 29 & & & $1 E+6$ & $1 \mathrm{E}+6$ \\
\hline $24 \mathrm{H}-2,0-5$ & 214 & 1,877 & 2,256 & 438.4 & 419 & & & $1 E+6$ & $1 E+6$ \\
\hline $25 \mathrm{H}-4,0-5$ & 222 & 6,333 & 28,803 & 622.5 & 3,640 & & & $1 \mathrm{E}+6$ & $1 \mathrm{E}+6$ \\
\hline $26 \mathrm{H}-2,0-5$ & 228 & 8.519 & 38,199 & $5,997.2$ & 49 & & & $1 E+6$ & $1 E+6$ \\
\hline $26 \mathrm{H}-4,0-5$ & 236 & 864 & 921 & $5,930.9$ & 1,025 & & & $1 E+6$ & $1 E+6$ \\
\hline $27 \mathrm{H}-5,0-5$ & 240 & 22,357 & 95,014 & $6,710.4$ & 77 & & & $1 E+6$ & $1 E+6$ \\
\hline $28 \mathrm{H}-2,0-5$ & 245 & 18,518 & 4,570 & $6,862.5$ & 3,327 & & & $\mathrm{IE}+6$ & $1 \mathrm{E}+6$ \\
\hline $29 \mathrm{H}-4,0-5$ & 256 & 3,231 & 7,809 & $4,120.9$ & 941 & & & $1 E+6$ & $1 \mathrm{E}+6$ \\
\hline $30 \mathrm{H}-1,145-150$ & 262 & 238,036 & & 2.447 .8 & 4,093 & & & $1 \mathrm{E}+6$ & $1 \mathrm{E}+6$ \\
\hline $30 \mathrm{H}-5,0-5$ & 267 & 2,434 & 4,466 & $6,238.6$ & 4,062 & & & $\mathrm{IE}+6$ & $1 \mathrm{E}+6$ \\
\hline $31 \mathrm{H}-6,0-5$ & 275 & 347,059 & & $3,969.2$ & 3,667 & & & $\mathrm{IE}+6$ & $1 \mathrm{E}+6$ \\
\hline $34 \mathrm{H}-1,127-130$ & 294 & 171.925 & & 8.586 .7 & 1.107 & & & $1 E+6$ & $1 E+6$ \\
\hline $34 \mathrm{H}-5,0-5$ & 298 & 1.174 & 1,136 & 8.967 .1 & 1,236 & & & $1 E+6$ & $1 \mathrm{E}+6$ \\
\hline $35 \mathrm{H}-4,0-5$ & 305 & 6.790 & 31,270 & 8.059 .9 & 2,129 & & & $1 E+6$ & $1 E+6$ \\
\hline $35 \mathrm{H}-5,0-5$ & 307 & 4.570 & 18,653 & $23,241.0$ & 2,955 & & & $1 E+6$ & $1 E+6$ \\
\hline $36 \mathrm{H}-2,65-70$ & 312 & & 1 & $4,123.0$ & 51 & & & $1 \mathrm{E}+6$ & $1 \mathrm{E}+6$ \\
\hline $37 \mathrm{H}-7,36-40$ & 329 & 409,891 & & $6,525.2$ & 3,070 & & & $1 \mathrm{E}+6$ & $1 \mathrm{E}+6$ \\
\hline $40 \mathrm{H}-3,0-5$ & 351 & 290,869 & & $68,043.0$ & 2,331 & & & $1 \mathrm{E}+6$ & $1 \mathrm{E}+6$ \\
\hline $41 X-1,0-5$ & 357 & 3,903 & 12,114 & 14.107 .0 & 56 & & & $1 \mathrm{E}+6$ & $1 \mathrm{E}+6$ \\
\hline $42 X-1,2-3$ & 367 & 126,046 & 29.812 & 950.1 & 1,581 & & & $1 \mathrm{E}+6$ & $1 \mathrm{E}+6$ \\
\hline $43 X-1,0-5$ & 376 & 20,811 & 89,179 & $33,329.0$ & 1,058 & & & $\mathrm{IE}+6$ & $1 E+6$ \\
\hline $44 X-3,145-150$ & 390 & 356.547 & & $20,264.0$ & 5,381 & & & $1 E+6$ & $1 E+6$ \\
\hline $45 X-2,0-5$ & 397 & 582,869 & & $8,309.5$ & 1,129 & & & $1 \mathrm{E}+6$ & $1 \mathrm{E}+6$ \\
\hline $47 X-1,0-2$ & 414 & 572.548 & & 10.031 .0 & 4,012 & & & $1 \mathrm{E}+6$ & $1 \mathrm{E}+6$ \\
\hline $48 x-1,0-5$ & 424 & 503.233 & & $28,517.0$ & 6,002 & & & $1 \mathrm{E}+6$ & $1 E+6$ \\
\hline $50 \times-1,20-24$ & 443 & 404,321 & & $5,017.1$ & 80 & & & $1 \mathrm{E}+6$ & $1 \mathrm{E}+6$ \\
\hline $52 X-1,20-25$ & 452 & 1,838 & 2,080 & 5.199 .9 & 68 & & & $\mathrm{IE}+6$ & $1 E+6$ \\
\hline $53 \times-1,48-53$ & 461 & 41 & 250 & $13,918.0$ & 6 & & & $1 \mathrm{E}+6$ & $1 E+6$ \\
\hline $54 X-2,0-5$ & 471 & 1,136 & 1,136 & $1,342.4$ & & & & $1 E+6$ & $1 E+6$ \\
\hline $55 X-1,0-5$ & 478 & 15,321 & 66,570 & $8,978.4$ & 1,888 & & & $1 \mathrm{E}+6$ & $1 \mathrm{E}+6$ \\
\hline $56 \mathrm{X}-1,40-45$ & 488 & 3.391 & 7,761 & 5.718 .5 & & & & $1 \mathrm{E}+6$ & $1 \mathrm{E}+6$ \\
\hline $57 X-1,137-140$ & 498 & 24,508 & 103,165 & 18.712 .0 & 62 & & & $1 \mathrm{E}+6$ & $1 E+6$ \\
\hline $58 \times-2,145-150$ & 508 & 510,48 & 208,69 & $24,246.6$ & 2 & & & $1 E+6$ & $1 \mathrm{E}+6$ \\
\hline $59 \mathrm{P}-1,0-5$ & 514 & 1,120 & 1,061 & $2,952.1$ & & & & $1 \mathrm{E}+6$ & $1 E+6$ \\
\hline $59 \mathrm{P}-1,47-48$ & 514 & 18,263 & 77,182 & 582.7 & 155 & & & $1 \mathrm{E}+6$ & $1 \mathrm{E}+6$ \\
\hline $60 \times-1,0-5$ & 515 & 4,150 & 13,898 & $19,026.0$ & & 1.0 & & 20,006 & 20,006 \\
\hline $61 X-1.145-150$ & 524 & 17.656 & 74.510 & 18.223 .0 & & 1.2 & & 15,123 & 15,123 \\
\hline $62 \times-2,0-5$ & 533 & 2,109 & 3,204 & $2,189.8$ & 37 & & & $1 \mathrm{E}+6$ & $1 \mathrm{E}+6$ \\
\hline $63 \times-2,0-5$ & 542 & 6,437 & 27,285 & $11,704.0$ & 2,616 & 1.4 & & 8.587 & 8,587 \\
\hline $64 X-2,0-5$ & 551 & 9,390 & 40.152 & 2.848 .0 & & 1.3 & & 2.243 & 2.243 \\
\hline $65 \times-4,145-150$ & 564 & 20,984 & 88,681 & $2,850.0$ & & 2.2 & 0.7 & 1,272 & 986 \\
\hline
\end{tabular}


Table 2. Vacutainer analyses for Hole 888B.

\begin{tabular}{|c|c|c|c|c|c|c|c|c|c|c|c|c|}
\hline $\begin{array}{l}\text { Core, section, } \\
\text { interval }(\mathrm{cm})\end{array}$ & $\begin{array}{l}\text { Depth } \\
\text { (mbsf) }\end{array}$ & $\mathrm{O}_{2}$ & $\mathrm{~N}_{2}$ & $\mathrm{C}_{1}$ & $\mathrm{CO}_{2}$ & $\mathrm{C}_{2}$ & $\mathrm{C}_{3}$ & $\mathrm{nC}_{4}$ & $\mathrm{nC}_{5}$ & $\mathrm{nC}_{6}$ & $\mathrm{C}_{1} / \mathrm{C}_{2}$ & $\mathrm{C}_{1} / \mathrm{C}_{2+}$ \\
\hline $31 \mathrm{H}-3,100-103$ & 271.82 & $2,851.6$ & 5,865 & 65.55 & 55.1 & 1.65 & 1.4 & 9.6 & 2.2 & 1.1 & $3.97 \mathrm{E}+1$ & 4 \\
\hline $36 \mathrm{H}-7,0-1$ & 319.01 & & & 50 & 373.2 & & & & & & $1.00 \mathrm{E}+6$ & $1 E+06$ \\
\hline $44 \mathrm{H}-3,38-39$ & 398.89 & 19,669 & 84,902 & 586,879 & 95 & 14.67 & 2.6 & & & & $4.00 \mathrm{E}+4$ & 33,983 \\
\hline $44 \mathrm{H}-4,143-144$ & 401.44 & 453,492 & 380 & 173,705 & 1600 & 3.4 & & & & & $5.11 E+4$ & 51,090 \\
\hline $62 \mathrm{H}-3,108-109$ & 535.79 & 27.4 & 163.3 & 450,158 & 9.1 & 23.57 & 1 & & & & $1.91 \mathrm{E}+4$ & 18,321 \\
\hline
\end{tabular}

Table 3. Total gas analyses for Hole $888 \mathrm{~B}$.

\begin{tabular}{lccc}
\hline $\begin{array}{c}\text { Core, section, } \\
\text { interval }(\mathrm{cm})\end{array}$ & $\begin{array}{c}\text { Depth } \\
\text { (mbsf) }\end{array}$ & $\begin{array}{c}\mathrm{CH}_{4}(\mathrm{ppb}) \\
\mathrm{ng} \mathrm{CH} / \mathrm{g} \\
\text { sediment }\end{array}$ & $\begin{array}{c}\delta^{13} \mathrm{CH}_{4} \\
(\% \circ)\end{array}$ \\
\hline 3H-2, 145-150 & 18 & 228 & -39.8 \\
$8 \mathrm{H}-4,140-150$ & 68.4 & 197 & -41.8 \\
$9 \mathrm{H}-3,140-150$ & 76.4 & 309 & -43.2 \\
$9 \mathrm{H}-3,140-150$ & 76.4 & 284 & -42.1 \\
$10 \mathrm{H}-4,140-150$ & 87.4 & 156 & -43.2 \\
$11 \mathrm{H}-3,142-152$ & 95.3 & 231 & -37.5 \\
$14 \mathrm{H}-4,138-150$ & 124 & 203 & -33.9 \\
$18 \mathrm{H}-4,135-150$ & 159 & 151 & -34.5 \\
$24 \mathrm{H}-1,135-150$ & 214.3 & 193 & -38.7 \\
$24 \mathrm{H}-1,135-150$ & 214.3 & 171 & -33.4 \\
$34 \mathrm{H}-2,130-150$ & 293.7 & 99 & -52.4 \\
$34 \mathrm{H}-2,130-150$ & 293.7 & 31 & -50.7 \\
$34 \mathrm{H}-2,130-150$ & 293.7 & 29 & -48.8 \\
$36 \mathrm{H}-2,70-80$ & 312.2 & 631 & -59.9 \\
$48 \mathrm{X}-\mathrm{CC}, 0-5$ & 423.5 & 350 & -57.4 \\
$61 \mathrm{X}-1,1-10$ & 522.8 & 409 & -50.3 \\
$63 \mathrm{X}-2,130-150$ & 543.2 & 253 & -50.6 \\
$63 \mathrm{X}-2,130-150$ & 543.2 & 414 & -51.7 \\
$65 \mathrm{X}-5,0-25$ & 564.1 & 204 & -50.4 \\
\hline
\end{tabular}

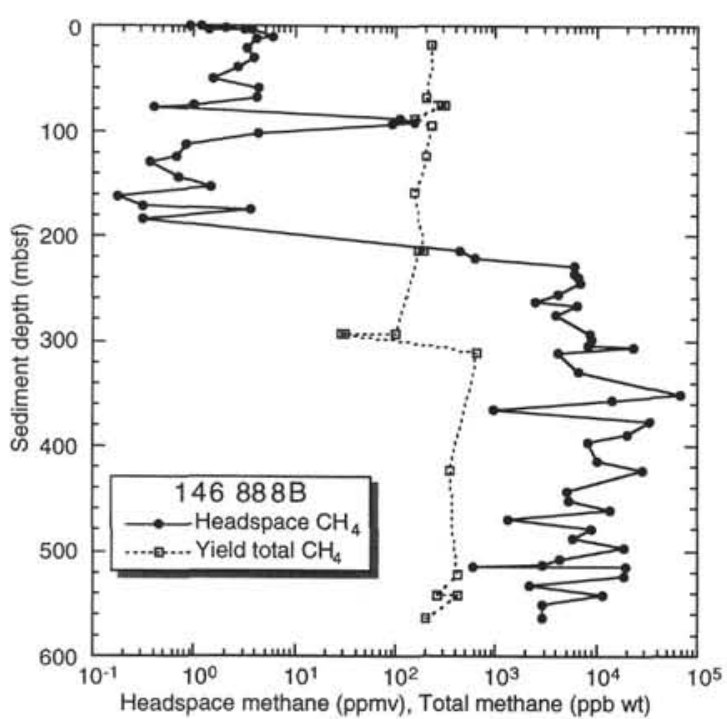

Figure 5. Depth distribution of headspace methane and total methane concentrations at Hole 888B.

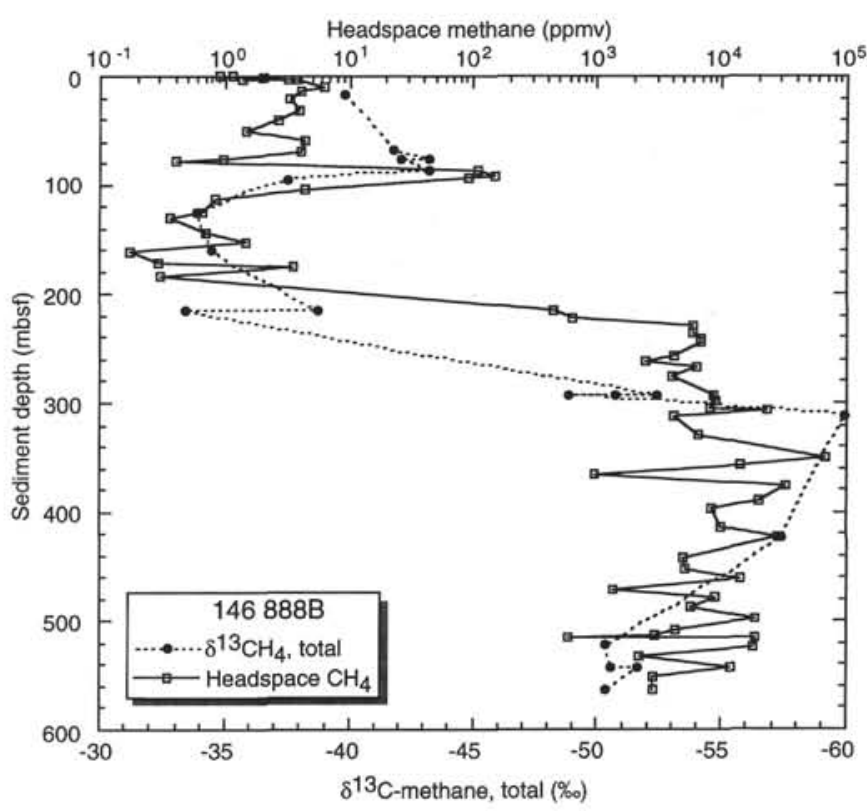

Figure 6. Depth distribution of total methane carbon isotope ratio and headspace methane concentrations at Hole 888B. 
Table 4. Headspace analyses for Hole 889A.

\begin{tabular}{|c|c|c|c|c|c|c|c|c|c|c|}
\hline $\begin{array}{l}\text { Core, section, } \\
\text { interval }(\mathrm{cm})\end{array}$ & $\begin{array}{l}\text { Depth } \\
\text { (mbsf) }\end{array}$ & $\mathrm{O}_{2}$ & $\mathrm{~N}_{2}$ & $\mathrm{C}_{1}$ & $\mathrm{CO}_{2}$ & $\mathrm{C}_{2}$ & $C_{3}$ & $\mathrm{iC}_{4}$ & $\mathrm{nC}_{4}$ & $\mathrm{C}_{1} / \mathrm{C}_{2+}$ \\
\hline IH-2, 0-6 & 21.5 & 19 & 114 & 58,980 & 1.2 & 1.4 & & & & 42,129 \\
\hline $1 \mathrm{H}-5,0-5$ & 26.0 & 96 & 585 & 60,149 & 3.6 & 1.5 & & & & 40,099 \\
\hline $2 \mathrm{H}-1,145-150$ & 31.0 & 1,295 & 1,231 & 50,134 & 2.2 & 2.4 & & & & 20,889 \\
\hline $2 \mathrm{H}-5,140-145$ & 36.9 & 94 & 577 & 31,452 & 4,386 & 1.3 & & & & 24,194 \\
\hline $3 \mathrm{H}-1,145-150$ & 40.5 & 353 & 713 & 30,622 & 3.27 & 1.6 & & & 1.9 & 8,749 \\
\hline $3 \mathrm{H}-6,6-10$ & 46.6 & 79 & 491 & 49,953 & 823.1 & 1.9 & & 3.6 & 0.9 & 7,805 \\
\hline $4 \mathrm{H}-3,0-5$ & 51.5 & 34.7 & 208 & 11,914 & 65.5 & 1.1 & & 9.0 & 0.9 & 10,831 \\
\hline $4 \mathrm{H}-6,0-5$ & 56.0 & 416 & 724 & 22,312 & 47.9 & 1.6 & & & & 13,945 \\
\hline $5 \mathrm{H}-4,0-5$ & 62.5 & 60 & 369 & 33,126 & 16 & 2.4 & & & & 13,803 \\
\hline $6 \mathrm{H}-6,0-5$ & 75.0 & 77.9 & 481 & 48,830 & 65.5 & 2.9 & & & & 16,838 \\
\hline $7 \mathrm{H}-2,0-5$ & 78.5 & 41.7 & 268 & 23,922 & 7.3 & 2.4 & & & & 9,968 \\
\hline $8 \mathrm{H}-4,0-5$ & 91.0 & 25 & 150 & 12,225 & 1.3 & 1.1 & & & & 11,114 \\
\hline $9 \mathrm{H}-4,0-5$ & 99.0 & 2,217 & 3,501 & 21,412 & 23.5 & 2.3 & & & & 9310 \\
\hline $10 \mathrm{H}-4,150-152$ & 110.0 & 3,661 & 9,217 & 9,652 & 39.3 & 2.2 & & & & 4,387 \\
\hline $11 \mathrm{H}-2,0-5$ & 115.0 & 6,093 & 26,875 & 27,858 & 1,662 & 4.6 & & & & 6,056 \\
\hline $12 \mathrm{H}-2,0-5$ & 120.5 & 4,143 & 13,472 & 9,652 & 1,921 & 2.2 & & & & 4,387 \\
\hline $13 \mathrm{H}-1,0-5$ & 127.0 & 9.055 & 40,702 & 76,498 & 1.634 & 14.1 & & & & 5,425 \\
\hline $14 \mathrm{H}-1,148-153$ & 129.5 & 8,356 & 36,951 & 10,053 & 1,770 & 11.2 & & & & 898 \\
\hline $15 \mathrm{P}-1,45-50$ & 129.5 & 2,103 & 3,089 & 51,184 & 1,898 & 33.4 & & & & 1,532 \\
\hline $17 X-2,75-80$ & 132.4 & 50,123 & 208,641 & 60,798 & 3,644 & 20.2 & 0.35 & & & 2,959 \\
\hline $18 \times-4,31-36$ & 144.4 & 58,023 & 237,429 & 46,432 & 6,325 & 12.5 & 1.66 & & & 3,279 \\
\hline $19 X-3,145-150$ & 153.6 & 25,010 & 109,405 & 48,534 & 1,923 & 19.25 & 0.55 & & & 2.451 \\
\hline $20 X-3,133-138$ & 163.0 & 33,101 & 140,520 & 54,756 & 1,758 & 12.6 & 0.6 & & & 4,148 \\
\hline $22 X-2,0-5$ & 179.0 & 4,255 & 15,273 & 30,784 & 1,383 & 9.5 & 0.7 & & & 3,018 \\
\hline $24 X-6,0-5$ & 195.3 & 4,061 & 12,777 & 47,330 & 3,792 & 22.3 & 3.4 & & & 1,842 \\
\hline $25 X-2,145-150$ & 200.3 & 3,965 & 11.814 & 48,662 & 3,185 & 16 & 1.7 & & & 2,749 \\
\hline $26 \times-3,0-5$ & 209.8 & 2.926 & 6,447 & 47,192 & 3,300 & 20.5 & 2.2 & & & 2,079 \\
\hline $28 \times-3,0-5$ & 220.3 & 3,748 & 10,249 & 71,222 & 2,035 & 33.3 & 2.7 & & & 1,978 \\
\hline $30 \times-3,0-5$ & 230.8 & 3,141 & 7.260 & 45,859 & 33,229 & 38.8 & 1.9 & & & 1,127 \\
\hline $31 \times-3,0-5$ & 240.2 & 1.986 & 2,669 & 74,882 & 3,294 & 35.9 & 1.3 & & & 2,013 \\
\hline $32 \mathrm{X}-1,125-130$ & 248.0 & 2,161 & 3,223 & 10,042 & & 12.78 & & & & 786 \\
\hline $32 \times-3,0-5$ & 249.7 & 60,347 & 249,019 & 10,055 & 982 & 12.15 & & & & 828 \\
\hline $34 X-2,145-150$ & 260.2 & 25,160 & 108,685 & 16,271 & 1.824 & 24.75 & 1.06 & & & 630 \\
\hline $36 \mathrm{X}-1,94-99$ & 267.7 & 184,462 & 860,777 & 23.299 & 10,299 & 35.66 & 1.7 & & & 624 \\
\hline $37 X-1,0-1$ & 275.2 & 2,522 & 4.859 & 34,004 & 2,449 & 35.3 & 0.59 & & & 947 \\
\hline $38 \mathrm{X}-1,0-1$ & 284.1 & 73,520 & 300,328 & 42,402 & 1,054 & 48.9 & 1.4 & & & 843 \\
\hline $39 X-1,45-50$ & 293.3 & 15,283 & 66,932 & 10,077 & 1,898 & 35.8 & 0.81 & & & 275 \\
\hline $40 \times-3,0-5$ & 301.5 & 4,173 & 14,104 & 25,619 & 1,811 & 58.3 & 0.72 & & & 434 \\
\hline $40 X-4,39-44$ & 301.5 & 3,298 & 7,850 & 26,463 & 2,487 & 134 & 1.6 & & & 195 \\
\hline $41 X-5,0-5$ & 310.5 & 7,872 & 34,327 & 38,529 & 1,124 & 83 & 1.1 & & & 458 \\
\hline $42 X-1,42-44$ & 319.5 & 51,386 & 212,776 & 8,929 & 963 & 108.9 & 1.6 & & & 81 \\
\hline $43 \times-2,0-5$ & 328.4 & 43,413 & 180,977 & 44,390 & 2,109 & 110.1 & 0.99 & & & 400 \\
\hline $44 X-1,101-106$ & 337.2 & 16,318 & 71,628 & 29,687 & 2,233 & 225.2 & 1.4 & & & 131 \\
\hline
\end{tabular}

Table 5. Vacutainer analyses for Hole 889A.

\begin{tabular}{|c|c|c|c|c|c|c|c|c|c|}
\hline $\begin{array}{l}\text { Core, section, } \\
\text { interval }(\mathrm{cm})\end{array}$ & $\begin{array}{l}\text { Depth } \\
\text { (mbsf) }\end{array}$ & Type & $\mathrm{C}_{1}$ & $\mathrm{C}_{2}$ & $\mathrm{C}_{3}$ & $\begin{array}{l}\text { Area } \\
(\mathrm{Vs})\end{array}$ & $\begin{array}{c}\delta^{13} \mathrm{CH}_{4} \\
(\% 6)\end{array}$ & $\begin{array}{c}\text { Area } \\
\text { (Vs) repl. }\end{array}$ & $\begin{array}{c}\delta^{13} \mathrm{CH}_{4} \\
(\% \circ)\end{array}$ \\
\hline IH-1, 41-42 & 20.4 & $\mathrm{~V}$ & $812,418.7$ & 31.8 & 0.3 & 8.6 & -84.4 & 12.0 & -83.6 \\
\hline $4 \mathrm{H}-2,132-133$ & 51.3 & V & $962,874.0$ & 65.8 & 0.6 & 16.3 & -66.2 & & \\
\hline $5 \mathrm{H}-7,37-38$ & 82.2 & $\mathrm{v}$ & $995,485.3$ & 83.5 & 0.6 & 19.9 & -61.5 & 12.9 & -61.5 \\
\hline $9 \mathrm{H}-8,91-92$ & 113.1 & V & 562.284 .0 & 62.7 & 1.5 & 8.8 & -57.1 & & \\
\hline $12 \mathrm{H}-5,5-6$ & 125.1 & $\mathrm{v}$ & $464,676.0$ & 211.7 & 1.0 & 9.0 & -56.7 & & \\
\hline $19 X-3,140-141$ & 144.5 & V & $782,283.9$ & 607.2 & 2.6 & 9.4 & -53.6 & & \\
\hline $20 \mathrm{X}-3,90-91$ & 162.5 & $\mathrm{v}$ & $969,920.4$ & 522.7 & 6.7 & 5.0 & -50.8 & & \\
\hline $22 X-6,28-29$ & 185.3 & $\mathrm{v}$ & $892,816.0$ & 866.9 & 9.2 & 5.3 & -49.9 & 7.6 & -50.4 \\
\hline $25 \mathrm{X}-2,100-101$ & 199.8 & v & $798,851.4$ & 687.4 & 7.7 & 7.3 & -50.7 & & \\
\hline $26 \times-4,56-57$ & 211.9 & $\mathrm{v}$ & 867.673 .0 & 625.4 & 6.5 & 7.0 & -50.3 & & \\
\hline $28 \mathrm{X}-4,101-102$ & 222.8 & V & $813,107.7$ & 867.3 & 5.1 & 13.0 & -51.0 & & \\
\hline $30 \times-2,81-82$ & 230.1 & V & $894,792.1$ & 775.7 & 4.1 & 11.5 & -53.7 & 10.6 & -53.8 \\
\hline $31 X-5,51-52$ & 243.7 & $\mathrm{~V}$ & $733,235.5$ & 412.2 & 3.7 & 10.0 & -54.9 & & \\
\hline $40 \mathrm{X}-1,102-103$ & 302.5 & V & 218.779 .0 & 206.1 & 1.0 & 8.0 & -49.5 & & \\
\hline $41 X-4,35-36$ & 315.4 & $\mathrm{v}$ & $860,362.1$ & $2,813.5$ & 5.0 & 14.5 & -47.4 & 9.5 & -47.3 \\
\hline 103/VAC7000004/1 & & Lab standard & $700,000.0$ & $87,500.0$ & $52,500.0$ & 8.7 & -36.4 & & \\
\hline 103/VAC7000004/2 & & Lab standard & $700,000.0$ & $87,500.0$ & $52,500.0$ & 13.8 & -36.6 & & \\
\hline $103 / \mathrm{VAC} 7000004 / 3$ & & Lab standard & $700,000.0$ & 87.500 .0 & $52,500.0$ & 7.2 & -37.1 & & \\
\hline 103/VAC7000004/4 & & Lab standard & 700.000 .0 & $87,500.0$ & $52,500.0$ & 11.3 & -36.5 & & \\
\hline 103/VAC $7000004 / 1$ & & Lab standard & $700,000.0$ & 87.500 .0 & $52,500.0$ & 8.7 & -36.4 & & \\
\hline 103/VAC7000004/2 & & Lab standard & $700,000.0$ & $87,500.0$ & $52,500.0$ & 13.8 & -36.6 & & \\
\hline
\end{tabular}

Note: repl. $=$ replicate analysis . 
Table 6. Total gas analyses for Hole 889A.

\begin{tabular}{|c|c|c|c|c|c|c|}
\hline $\begin{array}{c}\text { Core, } \\
\text { section, } \\
\text { interval }(\mathrm{cm})\end{array}$ & $\begin{array}{l}\text { Depth } \\
\text { (mbsf) }\end{array}$ & Type & $\begin{array}{l}\text { Weight } \\
(\mathrm{g}) \\
\text { frozen } \\
\text { sediment }\end{array}$ & $\begin{array}{l}\text { Area } \\
(\mathrm{Vs})\end{array}$ & $\begin{array}{c}\delta^{13} \mathrm{CH}_{4} \\
(\% \circ)\end{array}$ & $\begin{array}{c}\mathrm{CH}_{4} \text { (ppb wt) } \\
\mathrm{ng} \mathrm{CH}_{4} / \mathrm{g} \text { frozen } \\
\text { sediment }\end{array}$ \\
\hline $5 \mathrm{H}-1$ & 102 & VFG & 9.25 & 3.2 & -55.2 & 488 \\
\hline $5 \mathrm{H}-\mathrm{I}$ & 102 & VFG & 9.15 & 3.96 & -55.1 & 659 \\
\hline $13 \mathrm{H}-2$ & 129 & VFG & 8.18 & 15.45 & -56.8 & 2,241 \\
\hline $13 \mathrm{H}-2$ & 129 & VFG & 8.18 & 14.14 & -56.8 & 2,051 \\
\hline $24 X-5$ & 198.8 & VFG & 4.8 & 9.02 & -50.8 & 2,121 \\
\hline $24 X-5$ & 198.8 & VFG & 4.8 & 8.37 & -50.7 & 1,968 \\
\hline $32 \mathrm{X}-3$ & 248 & VFG & 7.89 & 2.03 & -54.2 & 271 \\
\hline $32 X-3$ & 248 & VFG & 7.89 & 1.87 & -53.5 & 250 \\
\hline $32 X-3$ & 248 & VFG & 6.85 & 1.8 & -52.9 & 290 \\
\hline $32 X-3$ & 248 & VFG & 6.85 & 1.64 & -52.8 & 265 \\
\hline $40 \times-4$ & 306.47 & VFG & 2.34 & 0.78 & -45.2 & 409 \\
\hline $43 \mathrm{X}-2$ & 329.8 & VFG & 12.57 & 1.08 & -48.0 & 95 \\
\hline $43 X-2$ & 329.8 & VFG & 12.57 & 2.65 & -47.3 & 232 \\
\hline
\end{tabular}

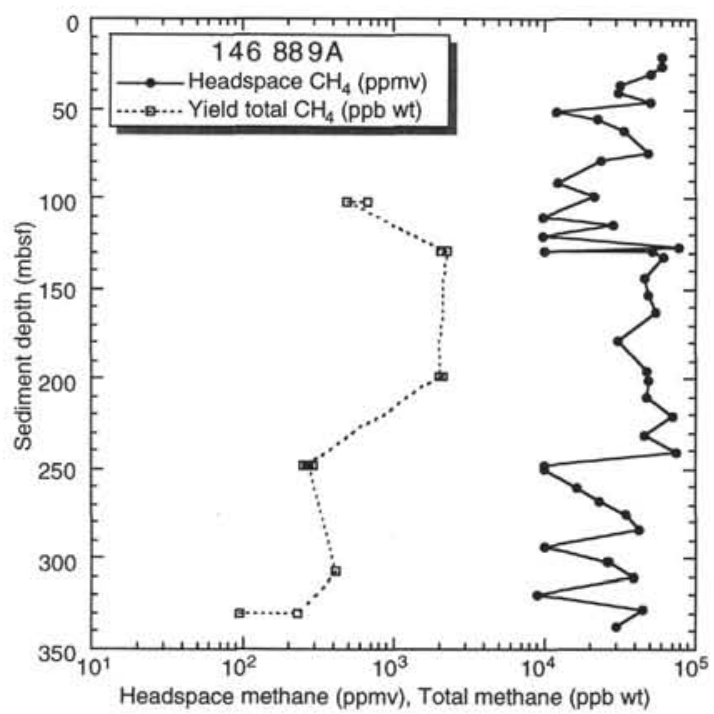

Figure 7. Depth distribution of headspace methane and total methane concentrations at Hole 889A.

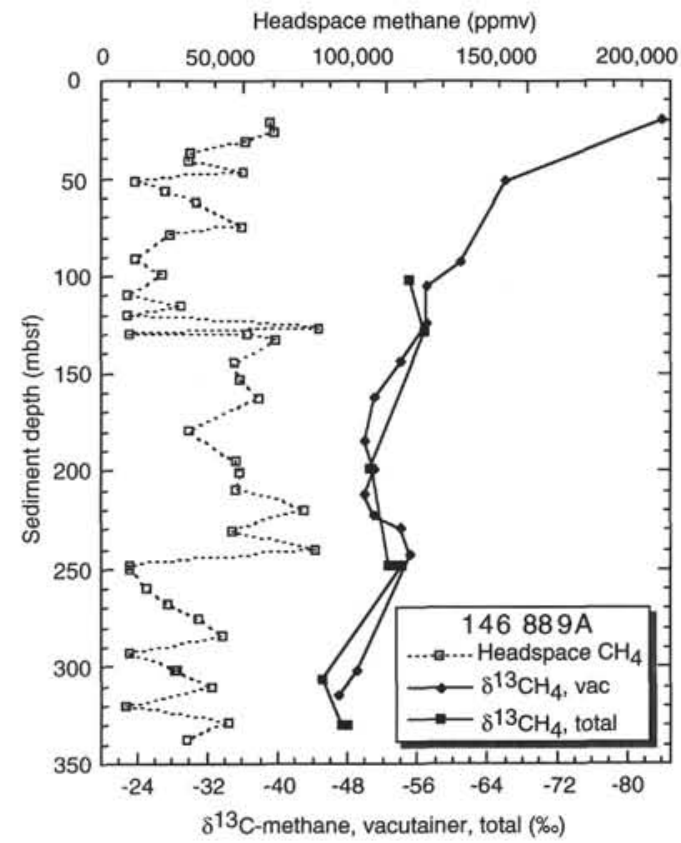

Figure 8. Depth distribution of Vacutainer and total methane carbon isotope ratio and headspace methane concentrations at Hole 889A. 
Table 7. Headspace and Vacutainer analyses for Holes 891A and B.

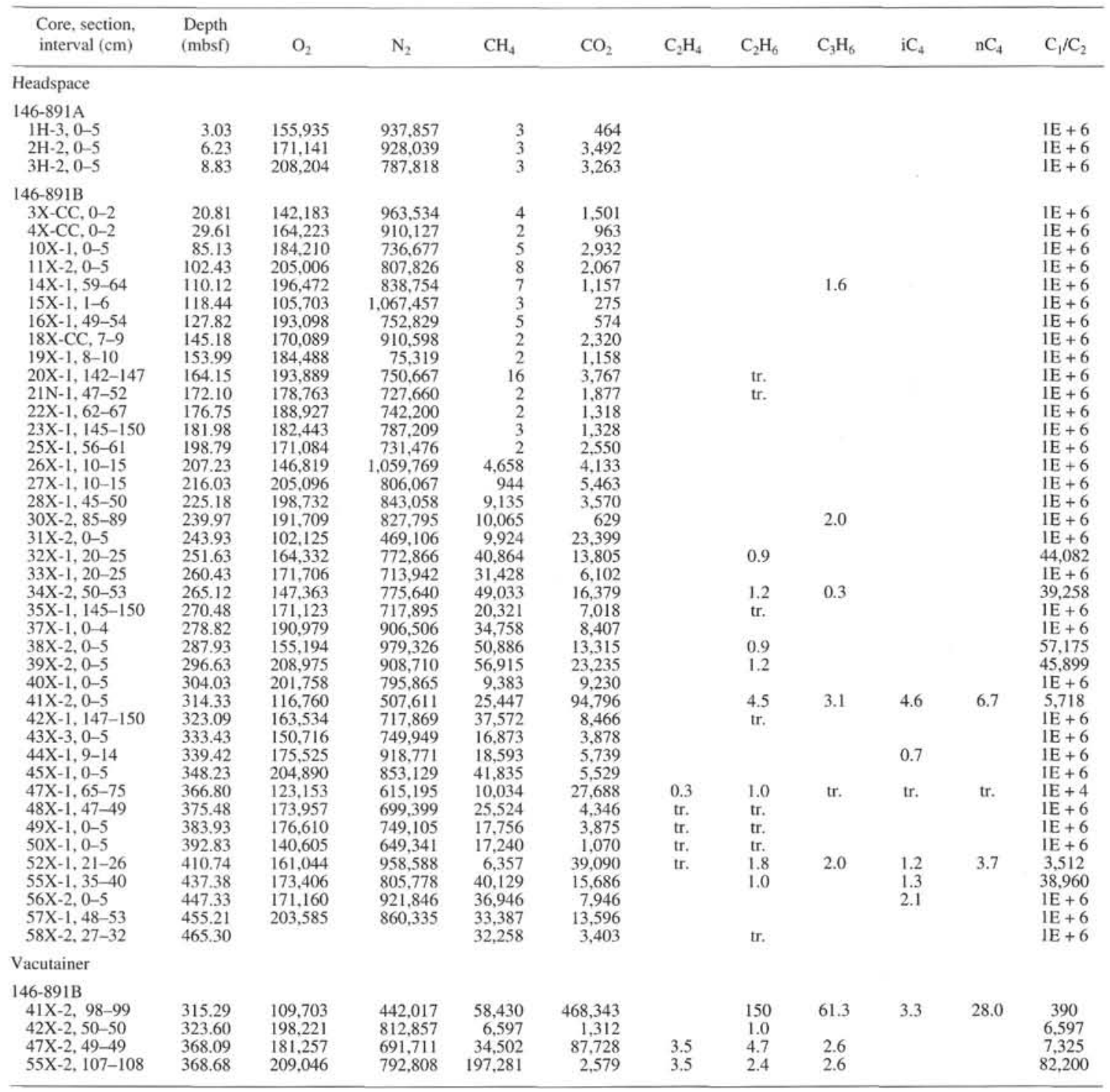

Table 8. Total gas analyses for Hole 891B.

\begin{tabular}{|c|c|c|c|c|c|c|}
\hline $\begin{array}{l}\text { Core, section, } \\
\text { interval }(\mathrm{cm})\end{array}$ & $\begin{array}{l}\text { Depth } \\
\text { (mbsf) }\end{array}$ & Type & $\begin{array}{l}\text { Weight } \\
(\mathrm{g}) \\
\text { frozen } \\
\text { sediment }\end{array}$ & $\begin{array}{l}\text { Area } \\
\text { (Vs) }\end{array}$ & $\begin{array}{c}\delta^{13} \mathrm{CH}_{4} \\
\text { (\%/o) }\end{array}$ & $\begin{array}{c}\mathrm{CH}_{4} \text { (ppb wt) } \\
\mathrm{ng} \mathrm{CH} / \mathrm{g} \\
\text { frozen sedimen }\end{array}$ \\
\hline $56 \mathrm{X}-1.72-89$ & 446.52 & VFG & 1.05 & 0.5377 & -50.7 & 563.1 \\
\hline $55 \mathrm{X}-2,130-150$ & 438.97 & VFG & 1.41 & 0.8087 & -63.4 & 659.5 \\
\hline $55 X-2,130-150$ & 438.97 & VFG & 1.41 & 0.7478 & -64.0 & 609.8 \\
\hline $52 X-1,7-21$ & 410.57 & VFG & 1.09 & 0.3444 & -38.7 & 427.7 \\
\hline $52 X-1,7-21$ & 410.57 & VFG & 1.09 & 0.3207 & -39.4 & 398.3 \\
\hline $43 X-1,10-27$ & 330.50 & VFG & 1.36 & 0.5119 & -44.2 & 446.9 \\
\hline $43 \times-1,10-27$ & 330.50 & VFG & 1.36 & 0.4754 & -42.9 & 415.0 \\
\hline $41 X-1,135-150$ & 314.15 & VFG & 0.81 & 0.3543 & -42.6 & 530.9 \\
\hline $21 \mathrm{~N}-\mathrm{CC}, 0-5$ & 172.12 & VFG & 1.64 & 0.2148 & -42.7 & 197.1 \\
\hline $15 X-1,82-90$ & 119.22 & VFG & 1.37 & 0.474 & -37.7 & 461.5 \\
\hline Standard & & $\mathrm{V}$ & & 14.5567 & -36.6 & \\
\hline Standard & & V & & 0.971 & -36.6 & \\
\hline
\end{tabular}

Note: VFG $=$ Vacutaine Free Gas, $\mathrm{V}=$ Vacutainer, and Vs $=$ GC/C/IRMS peak area mass 44 (Volt sec). 


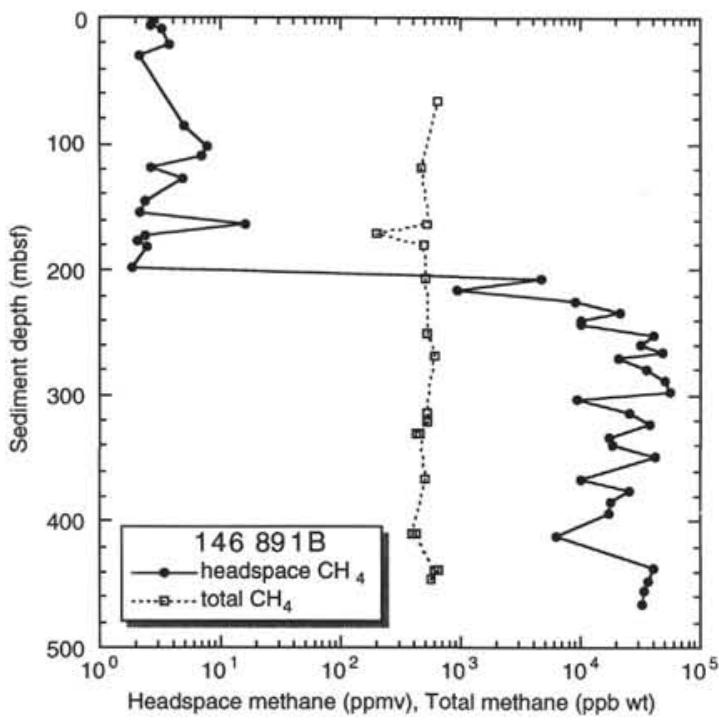

Figure 9. Depth distribution of headspace methane and total methane concentrations at Hole 891B.

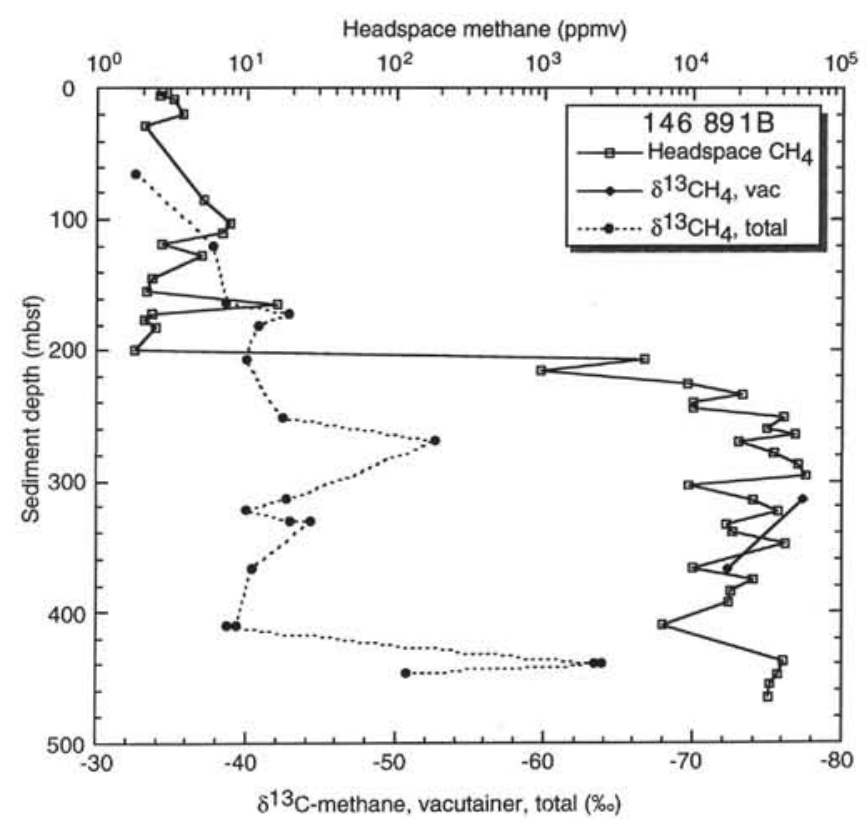

Figure 10. Depth distribution of total and Vacutainer methane carbon isotope ratios and headspace methane concentrations at Hole 891B. 
Table 9. Headspace analyses for Holes 892A, 892D, and 892E.

\begin{tabular}{|c|c|c|c|c|c|c|c|c|c|c|c|c|}
\hline $\begin{array}{l}\text { Core, section, } \\
\text { interval }(\mathrm{cm})\end{array}$ & $\begin{array}{l}\text { Depth } \\
\text { (mbsf) }\end{array}$ & $\mathrm{O}_{2}$ & $\mathrm{~N}_{2}$ & $\mathrm{CH}_{4}$ & $\mathrm{CO}_{2}$ & $\mathrm{C}_{2} \mathrm{H}_{4}$ & $\mathrm{C}_{2} \mathrm{H}_{6}$ & $\mathrm{H}_{2} \mathrm{~S}$ & $\mathrm{C}_{3} \mathrm{H}_{6}$ & $\mathrm{iC}_{4}$ & $\mathrm{nC}_{4}$ & $\mathrm{C}_{1} / \mathrm{C}_{2+}$ \\
\hline \multicolumn{13}{|l|}{$146-892 \mathrm{~A}$} \\
\hline $1 X-2,0-2$ & 1.51 & 161,918 & 692,267 & 80,252 & 1,713 & & 32 & tr & & & & 2,172 \\
\hline IX-4, 13-15 & 3.67 & 154,836 & 740,766 & 132,767 & 578 & & 77 & & & & & 1,718 \\
\hline $2 \times-3,0-5$ & 12.53 & 151,045 & 687,839 & 129,760 & 393 & & 53 & tr & & & & 2,442 \\
\hline $3 \mathrm{X}-1,0-5$ & 19.03 & 188,098 & 757,871 & 39,861 & 5,701 & & 10 & a & & & & 3,908 \\
\hline $3 \mathrm{X}-3,0-5$ & 22.03 & 176,566 & 783,687 & 33,947 & 1,684 & & 16 & & & & & 2,095 \\
\hline $4 X-1,95-100$ & 29.48 & 179,080 & 762,634 & 50,447 & 3,533 & & 34 & & & & & 1,497 \\
\hline $6 \mathrm{X}-1,145-150$ & 40.48 & 153,340 & 667,552 & 38,213 & 2,601 & tr. & 31 & & 0.8 & tr. & 1.8 & 1,063 \\
\hline $7 X-6,0-5$ & 56.03 & 127,472 & 698,926 & 58,799 & 9,700 & & 43 & & 1.9 & 4.5 & & 990 \\
\hline $8 X-3,145-150$ & 62.48 & 103,641 & 719,730 & 60,327 & 5,420 & 1.0 & 41 & & 6.8 & 5.1 & 3.4 & 884 \\
\hline $9 \mathrm{X}-1,0-5$ & 67.53 & 162,469 & 645,999 & 61,564 & 5,420 & & 281 & & 39.4 & 32.6 & 31.4 & 132 \\
\hline $9 \mathrm{X}-1,64-69$ & 68.17 & 114.746 & 710,498 & 10,015 & 5,420 & 0.9 & 339 & & 17.0 & 15.8 & 15.4 & 22 \\
\hline $11 \times-2,10-15$ & 79.63 & 143,192 & 704,554 & 35,800 & 334 & & 264 & & 35.8 & 38.3 & & 103 \\
\hline $12 X-1,60-65$ & 88.13 & 167,365 & 781,741 & 25,299 & 6,451 & tr. & 167 & & 29.4 & 20.4 & 2.6 & 106 \\
\hline $13 \mathrm{X}-3,96-101$ & 100.99 & 176,890 & $1,028,848$ & 20,081 & 4,741 & tr. & 121 & & 23.1 & 28.5 & tr & 107 \\
\hline $13 \mathrm{X}-8,0-5$ & 103.45 & 162,343 & $1,055,523$ & 61,304 & 4,849 & tr. & 186 & & 29.4 & 31.5 & 3.9 & 215 \\
\hline $14 \mathrm{X}-1,6-11$ & 106.59 & 173,101 & 718,681 & 32,385 & 1,147 & tr. & 249 & & 58.8 & 47.1 & 20.5 & 80 \\
\hline $14 \mathrm{X}-1,20-25$ & 106.73 & 147,248 & 781,741 & 12,530 & 2,941 & tr. & 327 & & 116.2 & 45.1 & 31.3 & 22 \\
\hline $15 \mathrm{X}-1,98-100$ & 116.99 & 146,993 & 686,294 & 47,617 & 11,138 & tr. & 354 & & 102.8 & 50.2 & 5.0 & 87 \\
\hline $16 \mathrm{X}-1,41-43$ & 125.92 & 191,269 & 925,261 & 19,513 & 4,060 & tr. & 525 & & 267.5 & 109.5 & 52.8 & 18 \\
\hline $17 \mathrm{X}-2,0-3$ & 135.61 & 207,599 & 808,947 & 61,211 & 3,056 & tr. & 302 & & 33.9 & 10.2 & 2.6 & 169 \\
\hline $18 X-2,0-5$ & 146.03 & 20 & (ס) & 11,856 & 3,056 & a. & 188 & & 50.4 & & & 50 \\
\hline $20 \mathrm{X}-\mathrm{CC}, 0-5$ & 167.07 & 147,042 & 734,007 & 18,868 & 2,680 & & 51 & & 18.1 & 19.7 & 8.3 & 148 \\
\hline $20 \mathrm{X}-2,145-150$ & 166.48 & 116,652 & 725,586 & 28,625 & 846 & & 106 & & 33.1 & 35.2 & 8.5 & 132 \\
\hline $21 X-1,7-12$ & 173.10 & 201,518 & 788412 & 95309 & 906 & & 176 & & 25.6 & 22.9 & 18.9 & 327 \\
\hline \multicolumn{13}{|l|}{$146-892 \mathrm{D}$} \\
\hline $1 X-1,5-7$ & 0.06 & 145,672 & 638,348 & 517 & 543 & & 5 & & 0.4 & & & 94 \\
\hline $2 X-1,0-5$ & 8.53 & 184,162 & 711,913 & 5,893 & 407 & & 1 & & 0.1 & & & 4,092 \\
\hline $2 \mathrm{X}-2,0-5$ & 10.03 & 121,472 & 697.491 & 70,147 & 932 & & 22 & & 0.1 & & & 3,248 \\
\hline $2 \mathrm{X}-2,145-150$ & 11.48 & 144,870 & 673,071 & 10,062 & 571 & & 16 & & 5.7 & 2.2 & 1.9 & 309 \\
\hline $3 X-1,0-5$ & 18.03 & 187,573 & 758,607 & 36,104 & 1,934 & & 15 & & 0.1 & & & 2,407 \\
\hline $3 \times-2,0-5$ & 19.53 & 123,895 & 596,837 & 18,222 & 2,353 & & 8 & & 0.1 & & & 2,159 \\
\hline $4 X-2,0-4$ & 29.02 & 197,011 & 774,283 & 14,399 & 3,716 & & 31 & & 0.6 & & & 449 \\
\hline $4 \mathrm{X}-3,30-35$ & 30.83 & 117,260 & 565,663 & 9.509 & 8,475 & & 6 & & tr. & & & 1,704 \\
\hline $4 \mathrm{X}-3,95-100$ & 31.48 & 194,465 & 781,034 & 8,306 & 4,097 & & 14 & & 0.4 & 2.5 & & 493 \\
\hline $5 \times-2,0-5$ & 38.53 & 203,576 & 787,825 & 46,883 & 4,492 & & 23 & & 0.6 & 2.5 & & 1,998 \\
\hline $5 X-3,0-5$ & 40.03 & 120,194 & 544,113 & 36,492 & 3,598 & & 39 & & 0.8 & & & 781 \\
\hline $6 \mathrm{X}-4,135-140$ & 52.38 & 160,326 & 666,856 & 24,069 & 4,070 & tr. & 29 & & 1.2 & & & 811 \\
\hline $7 X-4,145-150$ & 59.98 & 161,842 & 687,668 & 31,143 & 4,335 & & 29 & & 3.3 & 1.8 & & 874 \\
\hline $8 X-3,10-15$ & 64.83 & 163,236 & 790,548 & 10,078 & 1,975 & tr. & 17 & & 5.8 & 9.0 & 1.2 & 302 \\
\hline $9 \mathrm{X}-4,15-20$ & 73.98 & 125,804 & 634,234 & 38,891 & 4,546 & & 237 & & 31.3 & 41.0 & 5.1 & 114 \\
\hline $10 \times-4,15-20$ & 104.68 & 2,575 & 4,779 & 54.819 & 620 & & 385 & & 629.9 & 158.0 & 185.7 & 36 \\
\hline $10 \times-6,0-5$ & 107.53 & 60,632 & 260,612 & 43,753 & 125 & & 613 & & 576.0 & 191.3 & 400.0 & 22 \\
\hline $11 X-1,125-130$ & 110.78 & 54,405 & 249,574 & 54,511 & 3,233 & & 621 & & 101.0 & 10.9 & 3.0 & 71 \\
\hline $12 X-4,0-5$ & 123.53 & 153,879 & 696,886 & 55,436 & 2,893 & & 408 & & 76.6 & 36.7 & 2.7 & 97 \\
\hline $14 X-2,0-5$ & 139.53 & 206,460 & 851,797 & 42,269 & 3531 & & 109 & & 15.2 & 11.9 & & 300 \\
\hline $15 X-3,0-5$ & 150.53 & 135,574 & 638,314 & 65,074 & 963 & tr. & 269 & & 48.3 & 8.4 & & 194 \\
\hline $16 X-3,0-5$ & 160.03 & 142,266 & 614,633 & 106,913 & 5155 & tr. & 247 & & 30.8 & 19.2 & 3.1 & 345 \\
\hline \multicolumn{13}{|l|}{ I46-892E } \\
\hline $1 X-1,0-5$ & 0.03 & 42,504 & 249,592 & 730,754 & 3397 & tr. & 593 & & 0.3 & 0.9 & & 1,230 \\
\hline $1 \mathrm{X}-1,128-132$ & 1.30 & 99,929 & 703,422 & 43,950 & 7116 & tr. & 31 & & 0.6 & & & 1,384 \\
\hline $1 \mathrm{X}-2,125-130$ & 2.78 & 80,713 & 381,245 & 542,390 & 4257 & tr. & 448 & & 0.3 & 2.9 & & 1,202 \\
\hline $3 \mathrm{H}-5,125-130$ & 40.28 & 136,336 & 261,470 & 57.984 & 866 & tr. & 50 & & 0.3 & 3.3 & & 1,074 \\
\hline \multicolumn{13}{|c|}{ Headspace gases from hydrate dissociation in syringes } \\
\hline \multicolumn{13}{|l|}{$146-892 \mathrm{~A}$} \\
\hline $1 X-4,13-15$ & 3.67 & 178,369 & 872,556 & 66,408 & 504 & & 30 & 11,733 & 1.4 & & 21.2 & 1,182 \\
\hline $1 X-4,13-15$ & 3.67 & 152,440 & 598,181 & 10,210 & 711 & & 8 & 12,280 & 1.5 & 3.1 & 28.4 & 246 \\
\hline $1 X-4,13-15$ & 3.67 & 209,043 & 890,491 & 36,026 & 274 & & 12 & & & & & 3,116 \\
\hline $1 X-4,13-15$ & 3.67 & 165,080 & 747,892 & 46,085 & 731 & & 22 & 12,408 & 1.5 & & 31.0 & 840 \\
\hline
\end{tabular}


Table 10. Vacutainer analyses for Hole 892A.

\begin{tabular}{|c|c|c|c|c|c|c|c|c|}
\hline $\begin{array}{l}\text { Core, section. } \\
\text { interval }(\mathrm{cm})\end{array}$ & $\begin{array}{l}\text { Depth } \\
\text { (mbsf) }\end{array}$ & $\mathrm{CH}_{4}$ & $\mathrm{C}_{2}$ & $\mathrm{C}_{3}$ & $\begin{array}{l}\text { Area } \\
\text { (Vs) }\end{array}$ & $\begin{array}{c}\delta^{13} \mathrm{CH}_{4} \\
(\% \mathrm{oc})\end{array}$ & $\begin{array}{c}\text { Area } \\
\text { (Vs) repl. }\end{array}$ & $\begin{array}{l}\delta^{13} \mathrm{CH}_{4} \\
(\% \circ) \text { repl. }\end{array}$ \\
\hline $1 X-2,27-28$ & 1.78 & 802,197 & 807.9 & & 20.0 & -66.1 & 18.3 & -66.3 \\
\hline IX $-3,55-56$ & 3.55 & 805,749 & 818.5 & & 14.7 & -67.5 & & \\
\hline $2 X-4,114-115$ & 15.15 & 724,806 & 595.5 & & 19.2 & -66.3 & 8.9 & -66.5 \\
\hline $3 X-2,120-121$ & 21.71 & 919,909 & 359.0 & 10 & 8.3 & -66.3 & & \\
\hline $4 X-2,11-12$ & 30.12 & 455,003 & 677.6 & 0.6 & 12.3 & -67.9 & & \\
\hline $6 \times-2,74-74$ & 41.24 & 360,704 & 446.0 & 2.7 & & & 8.6 & -68.3 \\
\hline $6 \times-3,148-148$ & 43.48 & 851.695 & 993.7 & 6.1 & 9.4 & -66.2 & 21.3 & -66.0 \\
\hline $7 X-7,22-22$ & 57.72 & 854,304 & $2,070.0$ & 11.1 & 15.7 & -66.1 & & \\
\hline $8 X-4,135-135$ & 63.85 & 848,342 & $1,155.0$ & 32.6 & 14.5 & -66.4 & 10.1 & -66.1 \\
\hline $11 X-3,26-27$ & 81.26 & 931,873 & $6,322.0$ & 273.0 & 8.0 & -62.4 & 10.1 & -63.5 \\
\hline $13 \times-4,66-67$ & 102.17 & 949.757 & $5,310.9$ & 205.3 & 12.7 & -60.1 & 13.2 & -60.1 \\
\hline $15 X-1,84-84$ & 116.84 & 648,209 & $7,137.0$ & $3,473.0$ & 9.9 & -60.5 & 10.6 & -60.5 \\
\hline $17 X-2,63-63$ & 137.13 & 811,411 & 6.201 .0 & 332.2 & 11.6 & -62.3 & 20.0 & -61.2 \\
\hline $18 X-2,6-7$ & 146.07 & 139.571 & 352.9 & 29.4 & 5.6 & -61.8 & & \\
\hline $20 \mathrm{X}-1,147-148$ & 164.98 & 390,854 & $1,007.0$ & 102.0 & 8.8 & -62.1 & 8.1 & -62.5 \\
\hline G.HYDRATE-V.4 & & 800,000 & & & 0.2 & -64.8 & 0.5 & -64.1 \\
\hline 103/VAC900000PPM3/4 & Standard & 900,000 & $112,500.0$ & 67.500 .0 & 9.7 & -36.5 & & \\
\hline 103/VAC $900000 \mathrm{PPM} 3 / 1$ & Standard & 900,000 & $112,500.0$ & 67.500 .0 & 8.4 & -36.5 & & \\
\hline 103/VAC $900000 \mathrm{PPM} 3 / 2$ & Standard & 900,000 & $112,500.0$ & 67.500 .0 & 7.8 & -36.3 & & \\
\hline $103 / \mathrm{VAC} 900000 \mathrm{PPM} 3 / 3$ & Standard & 900,000 & 112.500 .0 & 67.500 .0 & 6.2 & -36.2 & & \\
\hline $103 / \mathrm{VAC} 300000 \mathrm{PPM} 7 / 16$ & Standard & 300,000 & 37.500 .0 & $22,500.0$ & 1.3 & -37.4 & & \\
\hline 103/VAC $900000 \mathrm{PPM} 2 / 5$ & Standard & 900,000 & $112,500.0$ & $67,500.0$ & 7.7 & -36.6 & & \\
\hline 103/VAC $900000 \mathrm{PPM} 2 / 6$ & Standard & 900,000 & $112,500.0$ & $67,500.0$ & 7.1 & -36.2 & & \\
\hline 103/VAC $300000 P P M 7 / 11$ & Standard & 300,000 & $37,500.0$ & 22.500 .0 & 8.3 & -36.7 & & \\
\hline $103 / \mathrm{VAC} 300000 \mathrm{PPM} 7 / 12$ & Standard & 300,000 & $37,500.0$ & 22.500 .0 & 7.1 & -39.4 & & \\
\hline 103/VAC $300000 \mathrm{PPM} 7 / 14$ & Standard & 300,000 & $37,500.0$ & 22.500 .0 & 3.5 & -36.6 & & \\
\hline 103/VAC $300000 \mathrm{PPM} 7 / 15$ & Standard & 300,000 & $37,500.0$ & 22.500 .0 & 3.6 & -36.6 & & \\
\hline
\end{tabular}

Note: repl. $=$ replicate analysis.

Table 11. Total gas analyses for Hole 892A.

\begin{tabular}{|c|c|c|c|c|c|c|}
\hline $\begin{array}{l}\text { Core, section, } \\
\text { interval }(\mathrm{cm})\end{array}$ & $\begin{array}{l}\text { Depth } \\
\text { (mbsf) }\end{array}$ & Type & $\begin{array}{c}\text { Weight } \\
(\mathrm{g}) \\
\text { frozen sediment }\end{array}$ & $\begin{array}{l}\text { Area } \\
\text { (Vs) }\end{array}$ & $\begin{array}{c}\delta^{13} \mathrm{CH}_{4} \\
(\% 0)\end{array}$ & $\begin{array}{c}\mathrm{CH}_{4}(\mathrm{ppb} \text { wt }) \\
\mathrm{ngCH} / \mathrm{g} \\
\text { frozen sediment }\end{array}$ \\
\hline $20 X-3,0-10$ & 166.5 & VFG & 3.45 & 7.1789 & -63.5 & 2,399 \\
\hline $20 \times-3,0-10$ & 166.5 & VFG & 3.45 & 6.5247 & -63.7 & 2,181 \\
\hline $20 X-3,0-10$ & 166.5 & VFG & 4.2 & 7.4953 & -63.8 & 2,252 \\
\hline $20 \times-3,0-10$ & 166.5 & VFG & 4.2 & 6.8528 & -63.9 & 2,059 \\
\hline $18 \times-1,140-150$ & 145.9 & VFG & 2.57 & 5.4744 & -64.0 & 2,763 \\
\hline $18 \mathrm{X}-1,140-150$ & 145.9 & VFG & 2.57 & 2.5609 & -63.5 & 1,293 \\
\hline $15 X-1,88-100$ & 116.9 & VFG & 1.55 & 1.4468 & -60.8 & 1,173 \\
\hline $13 \mathrm{X}-6,69-82$ & 101.6 & VFG & 2.13 & 3.4793 & -64.2 & 2,089 \\
\hline $11 \times-2,0-10$ & 79.5 & VFG & 1.83 & 5.0577 & -63.2 & 3,349 \\
\hline $9 X-1,69-74$ & 68.2 & VFG & 5.24 & 6.0181 & -63.8 & 1.356 \\
\hline $8 X-3,0-10$ & 61 & VFG & 0.94 & 0.9946 & -63.2 & 1,328 \\
\hline $8 X-3,0-10$ & 61 & VFG & 0.75 & 0.8715 & -63.5 & 1,335 \\
\hline $6 \times-2,0-10$ & 40.5 & VFG & 0.94 & 2.6292 & -65.9 & 3,574 \\
\hline $3 X-3,0-10$ & 21.8 & VFG & 1.1 & 0.6415 & -60.4 & 688 \\
\hline $2 X-3,0-11$ & 12.5 & VFG & 2.44 & 1.1539 & -61.9 & 572 \\
\hline $2 X-3,0-11$ & 12.5 & VFG & 2.23 & 1.3915 & -61.4 & 901 \\
\hline
\end{tabular}

